\title{
Enhanced recovery programme in gynaecology: outcomes of a hysterectomy care pathway
}

Debjani Mukhopadhyay, Khalil Razvi

Southend University NHS Foundation Trust

\begin{abstract}
There was a wide variation in the perioperative management of women undergoing hysterectomy for both benign and malignant disease at Southend University Hospital NHS Foundation Trust prior to 2010. The median length of stay following a hysterectomy and more radical gynaecological oncology surgery was five days and seven days respectively. The NHS East of England Strategic Health Authority commissioned the development of Enhanced Recovery Programme (ERP) in various surgical specialties including gynaecology and the pathway was implemented from 2012 onward.
\end{abstract}

Dedicated specialist nurses collected data prospectively. The median length of stay was shortened to three days. This difference was statistically significant with a $P$ value $=0.0001$. We describe the successful implementation of an ERP in Southend Hospital resulting with no difference in measurable morbidity and mortality, a shorter length of stay, and a high patient satisfaction scores and outcomes.

\section{Problem}

Southend University Hospital (SUH) NHS Foundation Trust is a cancer centre for gynaecological oncology and also provides care to women with general gynaecology problems. In 2010, data was collected from hospital episode statistics (HES) for mean and median length of stay following abdominal hysterectomy from all East of England Trusts. The result showed that the average length of stay (LOS) was five days at SUH while many of the other hospitals had a mean and median LOS for three days and two days respectively.[1] This resulted in a careful review of postoperative care of gynaecological patients at SUH.

\section{Background}

The Enhanced Recovery Programme (ERP) introduced by Kehlet et al, reduces morbidity and mortality in colorectal postoperative patients with a shortened hospital stay.[2,3] ERP has been extensively studied in patients undergoing colorectal surgery.[4] The implementation of ERP in gynaecological oncology involving a variety of complex pelvic and abdominal surgeries, remain sporadic with lack of randomised controlled trials (RCT) to advise either way.[5]

ERP provides consistency in preoperative, perioperative, and postoperative patient care with a well structured directive. It helps in preoperative optimization, preoperative counselling, and obtaining an informed consent. The patients can perceive realistic milestones, set their expectations, and plan around the surgery. The reduction in postoperative length of stay when following ERP is statistically significant and has a comparable rate of readmission when compared to traditional management.[6]

\section{Baseline measurement}

In 2010, Southend University Hospital did not have a documented care pathway for patients undergoing hysterectomy and more advanced abdominopelvic surgeries. There were wide variations in use of epidural, removal of catheter, commencement of feeding, and mobilisation. The median LOS was five days following an abdominal hysterectomy and seven days for more radical surgeries. The complication rate was $6 \%$ and readmission rate was $8 \%$.

\section{Design}

Southend University Hospital has a 20 bed gynaecological ward for all gynaecological emergencies and elective (benign and oncological) patients. A decision was made by the gynaecological oncology lead clinician to design a defined care pathway for postoperative patients. The "hysterectomy care pathway" would aim to ensure that patient is in the best possible physical state for surgery, to involve patients throughout their journey, improve care of postoperative patients, and get feedback from patients on their experience.

Literature search was conducted on care of postoperative patients.[7-9] Guidelines of other trusts with better performance were reviewed. Discussions were held with senior and experienced nursing staff to understand the local outlook on care of elective surgical patients at SUH.[10] Amalgamating the knowledge and experience, a "hysterectomy care pathway" was formulated. The principles adopted for care of elective gynaecological postoperative patients were as follows:

Preoperative:

- The preoperative optimization of patients was to be commenced in the community. This would involve optimal control of chronic diseases like asthma, diabetes, and improving nutritional status like correction of anaemia 


\section{BMJ Quality Improvement Reports}

- A dedicated preoperative clinic was set up for effective verbal counselling fortified by written information. The verbal and written information provided to patients was aimed to provide a better understanding of events to follow. This would help patients to set of clear expectations, realistic milestones, and effectively plan around surgery

- Mechanical bowel preparation was to be avoided to prevent dehydration and electrolyte disturbances.[11] Enemas were to be used sparingly as the relevant portion of the bowel would be cleared with less irritation to the bowel mucosa

- Preoperative fasting was set at six hours for food and two hours for clear fluids prior to surgery. This would prevent dehydration without increasing aspiration risks. Carbohydrate preloading was not deemed essential, as patient profile compared to colorectal patients were different

- Patients were to be assessed carefully before prescribing preanaesthetic medication to prevent delay in postoperative mobilisation

- All patients were admitted on the day of the surgery and antiembolism stockings or thromboembolic deterrent (TED) stockings were given.

Intraoperative:

- Minimally invasive surgery to be considered if possible[12]

- Nerve blocks and PCA were to be preferred for postoperative analgesia over epidural/spinal[13,14]

- Hypothermia was to be prevented by using warming blankets and warm fluids

- Single dose antibiotic was to be given before skin incision providing aerobic and anaerobic cover. Multiple doses of antibiotics are not known to provide any added benefit

- Nasogastric intubation and peritoneal drains were not to be used routinely to prevent delay in mobilisation[15,16]

- Use of transesophageal doppler for efficient fluid management was recommended as optimal fluid balance enhances recovery.[17]

Postoperative:

- Pain was to be controlled effectively for early mobilisation and epidural or patient controlled analgesia (PCA) and were to be gently weaned off to oral analgesia

- Oral intake of fluids and diet were to be commenced early to prevent insulin resistance and catabolic state and to build up an anabolic state for rapid recovery $[18,19]$

- Antiemetics were to be used effectively to prevent nausea and vomiting. Urinary catheters were to be removed in day one to aid mobilisation and minimise risk of infection

- lleus was to be prevented by avoiding opiates and using laxatives to promote gastric motility

- Mobilisation involving sitting out on the chair for eight hours in short periods throughout the day and walking up to 60 metres on the first day were to be implemented. The walking was to be increased to four times per day at least on subsequent days

- Correct use of thromboprophylaxis was to be ensured.

\section{Strategy}

A multiprofessional team was formed to design and implement the principles of ERP. The team consisted of consultant gynaecological oncologists, consultant anaesthetist, ERP nurse specialist, ward managers, oncology nurse specialists, dieticians, and physiotherapists. A document was drafted incorporating all the ERP principles modified to suit the gynaecological ward at SUH. The project was registered as an audit with the trust.

A booklet was designed that would follow the entire patient journey comprehensively.[20] Changes had been implemented in the preoperative, intraoperative, and postoperative care to improve patient journey and decrease hospital stay. The care of each patient was commenced on a booklet. The nurse led pre-op clinics had a structured printed pathway to perform preoperative checks on all patients. The anaesthetic and operative notes would document the specific intraoperative implementations brought on by ERP. Subsequent pages of the printed booklet continue to have a set postoperative documentation for each day. The booklet ensured the standard of care was maintained and errors minimized irrespective of the healthcare workers involved in patient care. During consultant ward rounds, juniors were instructed on steps to follow the care pathway and given feedback. If there is a delay on discharge or a complication it can be easily tracked back on the booklet and a cause can be identified.

The project received funding for an ERP nurse. There were two nurses sharing the job and responsibility. They work part time as ERP nurses and part time as ward nurses. Their role was to ensure the ERP principles were adhered to and prospective data collection. The gynaecological oncology consultant who implemented the project was notified of any deviation from the expected outcome.

Meetings were arranged every four months to discuss the comparative data. These quarterly meetings were aimed at core members speaking freely of their concerns and commendations.

In these meetings, each set of data collected in the preoperative, intraoperative, and postoperative columns for that quarter were analysed on a graph to see the trend and actual numbers. When the current data were compared to the previous data some useful insights were gained towards modifications and alterations that may contribute to an improved service.

There were two most important changes brought on by these 
meetings. One was to use PCA instead of epidurals. Mean day of mobilizing was a day late in women with epidurals than women with PCA. Initial pathway had high calorie preoperative drinks given to patients the day before similar to colorectal patients. The elderly patients tolerated it poorly and there was no difference in postoperative outcome between patients who had it and those who did not. The second change was to make high calorie preoperative drinks optional for patients undergoing complex surgery rather than routine practice.

The ERP nurses collected data prospectively for all patients undergoing major surgery or laparotomy for proven cancer or high suspicion of cancer by the gynaecological oncology team in 2012. In 2013, the project was rolled out to include all gynaecological major surgeries including benign cases. For this report, cases operated via laparoscopy and vaginal hysterectomies were excluded to present the changes in benign abdominal hysterectomies and more radical gynaecological oncological surgeries.

The demographic data collected were age at surgery. The preoperative data collected were BMI, ASA grade, and P-POSSUM score. The intraoperative data collected were procedure, length of surgery, measure of intravenous fluids given, and intraoperative complications. The postoperative data collected were on use of nasogastric tube, number of days until first bowel movement, use of antiemetic, days of epidural/PCA use, days of parenteral or oral analgesia use, days to remove urinary catheter, days to start diet, social care, postoperative complications, date of discharge, reason for delayed discharge, and readmissions within 30 days.

The primary outcome measures were length of hospital stay along with readmissions, and morbidity and mortality within 30 days. The ERP specialist nurse was to have a telephone conversation with the patient a few days after discharge. Patient outcome information was collated by distributing satisfaction questionnaires to patient upon discharge.

\section{Results}

The annual result of each year is presented separately to mark the improvement in the service (table 1). Clinical implementation of ERP commenced from 1st January 2012. The audit was conducted from the start date to 31st December 2013. The gynaecological oncology team operated on 101 patients in 2012. The eight patients who underwent laparoscopic surgeries or vulvectomies were not included. The rest of the 93 patients were included. The age range was 26 to 89 years and median age was 67 years.

140 patients were included out of the 255 patients been operated by the whole gynaecological team in 2013. The patients excluded had either vaginal or laparoscopic surgery. In 2013, the age range was 30 to 87 years and median age was 55 years.

In 2012, there were 39 (41.9\%) women with an American Society of Anesthesiologists (ASA) grade I, 34 (36.5\%) women with an ASA grade II, and 20 (21.5\%) women with an ASA grade III. In 2013, 113 $(80.7 \%)$ were ASA grade I, 15 (10.7\%) were ASA grade II, seven
(5\%) were ASA grade III. Five women did not have their ASA grade calculated.

12 patients did not have the body mass index (BMI) recorded in 2012. Among the rest 81 women, the mean BMI was 28 and the median BMI was 27. In 2013, 10 women did not have their BMI calculated. The mean BMI was 29 and median BMI was 27 .

In 2012, the gynaecological oncology team operated on all 93 cases. Of the 93 women, six women had bilateral salpingooophorectomy (BSO), 39 women had total abdominal hysterectomy and bilateral salpingo-oophorectomy ( $\mathrm{TAH}+\mathrm{BSO})$ with peritoneal washings, three women had radical hysterectomies, and 45 women had $\mathrm{TAH}+\mathrm{BSO}$ along with other procedures. These procedures were adhesiolysis, omental biopsy, infracolic omentectomy, lymph node dissections, apendicectomies, and bowel resection. In 2013, 44 cases were for benign indications involving BSO, unilateral salpingo-oophorectomy (USO) and $\mathrm{TAH}+\mathrm{BSO}$. The gynaecological oncology team operated on the remaining 96 cases for either suspected or proven malignancy. Of the 96 cases four women underwent radical hysterectomies, one woman was found to have unresectable disease and 91 women had laparotomies with radical debulking procedures involving omentectomy, appendicectomy, bowel resection, and lymph node dissection.

Three (3.2\%) patients received blood in 2012 and eight (5.7\%) patients received blood in 2013. Mean number of days until epidural or PCA were discontinued was two days in 2012 and 2013. One patient went into urinary retention in 2012 and no one failed trial without catheter (TWOC) in 2013. In 2012, the median of the number of days to discharge was four days. In 2013, the median of the number of days to discharge was three days.

In 2012, eight (8.6\%) patients had postoperative complications. The complications were relaparotomy for small bowel perforation, paralytic ileus, wound infection, first onset of atrial fibrillation and pneumonia, urinary retention, cardiac arrest for multiple pulmonary embolisms, haematuria in a patient on long term catheter preoperatively, and clostridium difficile infection during hospital stay. In 2013, five (3.6\%) patients endured postoperative complications. Three patients had bleeding more than expected and two patients had bowel injury.

In 2012, two (2.1\%) patients were readmitted. First woman was readmitted with pain and nausea, after four days. She was managed conservatively and discharged home in two days. The second patient was readmitted after two days and had a relaparotomy for delayed bowel injury. In 2013, there were five (3.5\%) readmissions within 30 days. The median days of readmissions was 7.5 days ( 5 to 18 days range). The indications for readmissions were wound dehiscence requiring return to theatre for resuturing of rectus sheath, wound infection that required return to theatre for debridement, pelvic haematoma, pain owing to sarcoma, and bleeding per vaginum. The patient with sarcoma was the only mortality during the two years of ERP audit. The patient was transferred to a specialist sarcoma unit for further treatment and died within 30 days of initial surgery. 
In 2012, two patients with ovarian cancer had unplanned admission to ITU. One case was for a delayed bowel complication that required a second laparotomy and the other case was for major pulmonary embolism.

In 2013, three patients had unplanned admission to critical care. First case was after secondary haemorrhage requiring a second laparotomy following a benign hysterectomy. The second case required HDU admission for more than anticipated complex debulking surgery for a sarcoma. The last patient, an 82 year old lady was admitted to ITU following postoperative pneumonia.

In 2012, the median of the number of days to discharge was four days. In 2013, the median of the number of days to discharge was three days. This compares very favourably to 5.0 days that was the median number of days in 2010 before the ERP programme was implemented. There were 45/101 (44.6\%) cases of malignancy in 2012 and $26 / 140$ (18.5\%) cases of malignancy in 2013 . The rest of the cases had a benign histology.

The decrease in median LOS from five days to three days in postoperative patients was highly statistically significant $(P=0.0001)$. The cost of bed per day in gynaecological ward is $£ 286.00$.

Reducing the LOS by two days for each postoperative patient would have saved over $£ 80,000.00$ if applied to all 140 patients in 2013.

Patient satisfaction questionnaires were given to patients upon discharge from the ward in 2013. 100 (71.5\%) questionnaires were returned. Of the 100 , about $75 \%$ of the patients answered positively and less than $5 \%$ were either unsure or replied negatively to the questions (table 2).

See supplementary file: ds5147.pdf - "Outcomes of hysterectomy care pathway; patient satisfaction questionnaires in 2013 (100/140)"

\section{Lessons and limitations}

"Hysterectomy care pathway" was successfully implemented owing to a coherent multidisciplinary team effort involving nursing care, anaesthetic/pain team, physiotherapists, dieticians, and the surgical team. The implementation was less daunting as some principles of "hysterectomy care pathway" were already in practice and the nurses were familiar to them. The "hysterectomy care pathway" organised the thought process and explained the rationale better. This helped the care providers to work with the care pathway.

A protocol itself is not a fail-safe mechanism [20] and the introduction of the booklet aided the juniors and new members of staff to deliver the expected standard of care. The prospective data collection was an effective tool to audit the service. Quarterly reviews of outcomes identified challenges early to improvise on the current care. Positive feedback from patients with satisfaction questionnaires stipulated evidence that of the "hysterectomy care pathway" was effective, acceptable, and offered encouragement to all care providers.

The significant reduction in gynaecological oncology cases in 2013 was because the vast majority of endometrial cancer cases being performed laparoscopically compared to 2012. Despite best efforts, there will be exceptions where discharge will be delayed and a realistic individualised discharge date may be helpful to prevent disappointment.

One limitation of our project may be the Hawthorne effect. Every staff member was aware of the 'hysterectomy care pathway' and prospective data collection. The new found enthusiasm of staff may be reflected in our project outcome. That said, continuous practice of the hysterectomy care pathway for two years has ingrained it as the routine practice for care offered to gynaecological operative patients at Southend Hospital. As mentioned earlier, the printed booklet ensures the continued care taking into account frequent incorporation of new staff at NHS hospitals. The funding for the ERP nurses are ongoing so prospective data collection has continued. There is still active input from oncology consultants and all deviations from outcome are assessed for quality control of the care offered to patients.

\section{Conclusion}

Our results, having followed the "hysterectomy care pathway" for two years provide evidence that ERP correctly adopted in gynaecological surgery reduces hospital stay and morbidity without any increase in readmissions. The LOS decreased by two days in women undergoing gynaecological hysterectomies or more radical open abdominal surgeries. This was found to be statistically significant. An evidence based protocol and a care pathway booklet were imperative for the above outcome. Decrease in bed occupancy saves $£ 286.00$ per day per patient. Apart from economic benefits there was documented evidence of patient satisfaction and acceptability.

\section{References}

1. Razvi K, Spencer C. Enhanced Recovery Programme Guideline; Vaginal , abdominal and laparoscopic hysterectomy. Commissioned by NHS East of England Enhanced Recovery Committee. December 2011; 1-16.

2. Kehlet $\mathrm{H}$, Dahl JB. Anaesthesia, surgery, and challenges in postoperative recovery. Lancet 2003 Dec 6 ;362(9399):1921-8. Available from: http://www.ncbi.nlm.nih.gov/pubmed/14667752.

3. Kehlet $\mathrm{H}$, Wilmore DW. Evidence-based surgical care and the evolution of fast-track surgery. Ann Surg 2008 Aug ;248(2):189-98. Available from: http://www.ncbi.nlm.nih.gov/pubmed/18650627.

4. Gouvas N, Tan E, Windsor A, Xynos E, Tekkis PP. Fasttrack vs standard care in colorectal surgery: a meta-analysis update. Int J Colorectal Dis 2009 Oct;24(10):1119-31. Available from: http://www.ncbi.nlm.nih.gov/pubmed/19415308.

5. Lu D, Wang X, Shi G. Perioperative enhanced recovery programmes for gynaecological cancer patients. Cochrane database Syst Rev 2012 Jan;12:CD008239. Available from: http://www.ncbi.nlm.nih.gov/pubmed/23235656.

6. Teeuwen PHE, Bleichrodt RP, Strik C, Groenewoud JJM, 
Brinkert W, van Laarhoven CJHM, et al. Enhanced recovery after surgery (ERAS) versus conventional postoperative care in colorectal surgery. J Gastrointest Surg 2010 Jan;14(1):88-95. Available from: http://www.pubmedcentral.nih.gov/articlerender.fcgi?artid=2 793377\&tool=pmcentrez\&rendertype =abstract

7. Dickson E, Argenta PA, Reichert JA. Results of introducing a rapid recovery program for total abdominal hysterectomy. Gynecol Obstet Invest 2012 Jan;73(1):21-5. Available from: http://www.ncbi.nlm.nih.gov/pubmed/22156551.

8. Chase DM, Lopez S, Nguyen C, Pugmire GA, Monk BJ. A clinical pathway for postoperative management and early patient discharge: does it work in gynecologic oncology? Am J Obstet Gynecol 2008 Nov;199(5):541.e1-7. Available from: http://www.ncbi.nlm.nih.gov/pubmed/18513685.

9. Marx C, Rasmussen T, Jakobsen $\mathrm{DH}$, Ottosen $\mathrm{C}$, Lundvall L, Ottesen B, et al. The effect of accelerated rehabilitation on recovery after surgery for ovarian malignancy. Acta Obstet Gynecol Scand 2006 Jan;85(4):488-92. Available from: http://www.ncbi.nlm.nih.gov/pubmed/16612713.

10. Sjetne IS, Krogstad U, Ødegård S, Engh ME. Improving quality by introducing enhanced recovery after surgery in a gynaecological department: consequences for ward nursing practice. Qual Saf Health Care 2009 Jun;18(3):236-40. Available from: http://www.ncbi.nlm.nih.gov/pubmed/19468009.

11. Güenaga KF, Matos D, Wille-Jørgensen P. Mechanical bowel preparation for elective colorectal surgery. Cochrane database Syst Rev 2011 Jan;(9):CD001544. Available from: http://www.ncbi.nlm.nih.gov/pubmed/21901677.

12. Nieboer TE, Johnson N, Lethaby A, Tavender E, Curr E, Garry R, et al. Surgical approach to hysterectomy for benign gynaecological disease. Cochrane database Syst Rev 2009 Jan;(3):CD003677. Available from:

http://www.ncbi.nlm.nih.gov/pubmed/19588344

13. Borendal Wodlin N, Nilsson L, Kjølhede P. The impact of mode of anaesthesia on postoperative recovery from fasttrack abdominal hysterectomy: a randomised clinical trial. BJOG 2011 Feb;118(3):299-308. Available from: http://www.ncbi.nlm.nih.gov/pubmed/20840529

14. Wodlin NB, Nilsson L, Arestedt K, Kjølhede P. Mode of anesthesia and postoperative symptoms following abdominal hysterectomy in a fast-track setting. Acta Obstet Gynecol Scand 2011 Apr;90(4):369-79. Available from: http://www.ncbi.nlm.nih.gov/pubmed/21332679

15. Lopes AD, Hall JR, Monaghan JM. Drainage following radical hysterectomy and pelvic lymphadenectomy: dogma or need? Obstet Gynecol 1995 Dec;86(6):960-3. Available from: http://www.ncbi.nlm.nih.gov/pubmed/7501348.

16. Nelson R, Edwards S, Tse B. Prophylactic nasogastric decompression after abdominal surgery. Cochrane database Syst Rev 2007 Jan;(3):CD004929. Available from: http://www.ncbi.nlm.nih.gov/pubmed/17636780.

17. Kuper M, Gold SJ, Callow C, Quraishi T, King S, Mulreany $A$, et al. Intraoperative fluid management guided by oesophageal Doppler monitoring. BMJ 2011 Jan;342:d3016. Available from: http://www.ncbi.nlm.nih.gov/pubmed/21610051.
18. Charoenkwan K, Matovinovic E. Early versus delayed oral fluids and food for reducing complications after major abdominal gynaecologic surgery. Cochrane database Syst Rev 2014 Jan;12:CD004508. Available from: http://www.ncbi.nlm.nih.gov/pubmed/25502897

19. Minig L, Biffi R, Zanagnolo V, Attanasio A, Beltrami C, Bocciolone L, et al. Early oral versus "traditional" postoperative feeding in gynecologic oncology patients undergoing intestinal resection: a randomized controlled trial. Ann Surg Oncol 2009 Jun;16(6):1660-8. Available from: http://www.ncbi.nlm.nih.gov/pubmed/19330379.

20. Maessen J, Dejong CHC, Hausel J, Nygren J, Lassen K, Andersen $\mathrm{J}$, et al. A protocol is not enough to implement an enhanced recovery programme for colorectal resection. $\mathrm{Br} \mathrm{J}$ Surg 2007 Feb;94(2):224-31. Available from: http://www.ncbi.nlm.nih.gov/pubmed/17205493.

\section{Declaration of interests}

Nothing to declare.

\section{Acknowledgements}

We are very grateful to the following group and individuals at Southend University Hospital NHS Foundation Trust:

- SUH ERP care pathway group

- Mrs Anne Cunnell for data collection

- Miss Ellie Cole for statistical support

- Mr Christopher Spence for data analysis

- Dr Zoey Keeble for data collection.

Correction notice: this article has been corrected since it was first published. Khalil Razvi has been added as an author

\section{Ethical approval}

The work was deemed an improvement study and not a study on human subjects and ethical approval was not required as per local policy. 